\title{
Nuclear Self-energy and Realistic Interactions
}

\author{
T. Frick, Kh. Gad, H. Müther \\ Institut für Theoretische Physik, \\ Universität Tübingen, D-72076 Tübingen, Germany \\ P. Czerski \\ Institute Fiz. Jadrowej, Pl-31-342 Krakow, Poland
}

\begin{abstract}
The structure of nucleon self-energy in nuclear matter is evaluated for various realistic models of the nucleon-nucleon (NN) interaction. Starting from the Brueckner-Hartree-Fock approximation without the usual angle-average approximation, the effects of hole-hole contributions and a self-consistent treatment within the framework of the Green function approach are investigated. Special attention is paid to the predictions for the spectral function originating from various models of the $\mathrm{NN}$ interaction which all yield an accurate fit for the NN phase shifts.
\end{abstract}

\section{INTRODUCTION}

One of the central issues of quantum many-body theory and theoretical nuclear physics is the attempt to derive the bulk properties of nuclear systems from realistic models of the nucleon-nucleon $(\mathrm{NN})$ interaction. Various approximation schemes have been developed to describe the correlations which are induced into the many-nucleon wave function by the strong short-range and tensor components of a such a realistic NN force. For a recent review on such methods see e.g. [1,2]. One of the most popular approximation schemes, which is frequently used in nuclear physics, is the hole-line expansion, in particular the approximation of lowest order, the Brueckner-Hartree-Fock (BHF) approximation.

In this approach the NN correlation are taken into account in solving the Bethe-Goldstone equation, which leads to the so-called G-matrix. The G-matrix accounts for multiple scattering processes and therefore corresponds to the NN scattering matrix $T$. In contrast to the Lippmann-Schwinger equation leading to $T$ the Bethe-Goldstone equation accounts for effects of the nuclear medium: The propagator for the intermediate two-particle states is restricted to particle states, i.e. to single-particle states with energies above the Fermi energy $\varepsilon_{F}$, and is defined in terms of the single-particle energies for the nucleons in the medium. This choice of the single-particle energies in the propagator of the Bethe-Goldstone equation is motivated by the Bethe-Brandow-Petschek theorem [3].

Strictly speaking, however, the Bethe-Brandow-Petschek theorem only defines the energy variable to be used in the calculation of self-energy or single-particle potential for the hole states. The choice for the propagator of the particle states is not defined on this level of the hole-line expansion and therefore has been discussed in a controversial way. The conventional choice has been to ignore self-energy contributions for the particle states completely and approximate the energies by the kinetic energy only. This conventional choice is sup- 
ported by the $S_{2}$ approximation of the coupled cluster or exponential S method [ब, which essentially leads to the same approach, This conventional choice for the single-particle spectrum, however, is not very appealing as it leads to a gap at the Fermi surface: the propagator for single-particle states with momenta below the Fermi momentum $k_{F}$ is described in terms of a bound single-particle energy while the corresponding spectrum for the particle states starts at the kinetic energy for the momentum $k_{F}$.

Mahaux and collaborators [5] argued that it would be more natural to choose the propagator according to the Green function method, i.e. define the single-particle propagator with a single-particle energy which includes the real part of the self-energy as a single-particle potential for particle and hole states. This leads to a spectrum which is continuous at the Fermi momentum, which provided the name "continuous choice" for this approach. This continuous choice leads to an enhancement of correlation effects in the medium and tends to predict larger binding energies for nuclear matter than the conventional choice.

The optimal choice for the single-particle spectrum seems to be in between the conventional and the continuous choice spectrum. This has been demonstrated by the recent investigations of the Catania group [6]. They calculated the effects of contributions in the hole line expansion originating from three-hole line terms [7] using both the conventional and the continuous choice spectrum. The inclusion of the three-hole line terms leads to a calculated binding energy of nuclear matter which is in between the results of BHF calculation with the conventional and the continuous choice and rather insensitive to the choice made. The attractive contribution obtained from the three hole line terms using the conventional choice is larger than the repulsive contribution evaluated with the continuous choice. This demonstrates that the continuous choice yields a slightly better convergence than the conventional one. The results also indicate that the two-nucleon correlations tend to be underestimated with the conventional choice and slightly overestimated with the continuous choice for the single-particle spectrum. It is one of the aims of this manuscript to explore the role of the single-particle propagator in the evaluation of NN correlation in more detail.

In solving the Bethe-Goldstone equations and its extensions to the three hole line terms, it has been common practise to use an approximation for the Pauli operator which is independent on the angle between the center of mass momentum of the interacting pair of nucleons and its relative momentum. Using the angle-average approximation and assuming that the single-particle spectrum can be parametrised as function of the momentum in a quadratic form, one can solve the Bethe-Goldstone equation easily using a partial wave expansion. During the last few years methods have been developed to solve the Bethe-Goldstone equation without this angle-average approximation [8.9]. It turns out that the results are not very sensitive to the treatment of the Pauli operator but they change in a non-negligible way if a single-particle spectrum is used, which differs from a simple quadratic parametrisation. This has recently been observed by Baldo and Fiasconaro [10 using an angle-average approach. We are going to explore this feature in more detail without using the angle-average approximation.

The continuous choice for the single-particle spectrum has been motivated from the method of self-consistent Green functions in the many-body theory. Within this scheme, however, the evaluation of the self-energy is done treating particle-particle and hole-hole ladders at the same level of the approximation scheme replacing the Bethe-Goldstone equation by the corresponding Galitzkii-Feynman equation. If one tries to perform such calculations for 
realistic NN forces, one encounters the problem of the so-called pairing instabilities [11-13]. These pairing effects can be taken into account by means of the BCS approach [14 [16]. At the empirical saturation density of symmetric nuclear matter the solution of the gap equation in the ${ }^{3} S_{1}-{ }^{3} D_{1}$ partial wave leads to an energy gap of around $10 \mathrm{MeV}$. Another approach is to consider an evaluation of the generalised ladder diagrams with "dressed" single-particle propagators. This means that the single-particle Green functions are not approximated by a mean-field approach but consider single-particle strength distributed over all energies. Various attempts have been made in this direction, considering a parametrisation of the single-particle Green function in terms of various poles [17], employing simplified (separable) interaction models [13] or considering the case of finite temperature.

The same instabilities also occur in studies of finite nuclei [18], leading to divergent contributions to the binding energy from the generalised ring diagrams. These contributions remain finite if the single-particle propagators are dressed in a self-consistent way.

Here we want to explore some features of the single-particle self-energy and the corresponding Green function for infinite symmetric nuclear matter at temperature $T=0$. As a starting point we consider the BHF approximation employing a self-consistent continuous choice spectrum without angle-averaging. The contribution of hole-hole ladder terms are then added in a perturbative approach. We then analyse the energy distribution of the spectral single-particle strength and define appropriate mean values. These mean values are used to calculate the self-energy in a self-consistent way.

These studies are performed for various NN interactions and we will pay special attention to the differences in the properties of the nucleon self-energy which can be related to the interaction model. In particular, we will consider local and non-local models of the NN interaction, which have been defined by the groups in Moscow (Idaho) [19], Argonne [20], and Nijmegen [21]. All these interaction models yield a very accurate fit to a selected database of NN scattering data. For further comparison we will also consider two older version of the Bonn potential, defined in [22].

After this introduction we will discuss some features of the self-consistent BHF approach

for the self-energy in section 2. The effect of the hole-hole terms in the self-energy and a self-consistent treatment of the single-particle Green function will be presented in section 3 . The main conclusions are summarised in the final section.

\section{SINGLE PARTICLE SPECTRUM IN THE BHF APPROXIMATION}

The self-energy or single-particle energy of a particle in the Brueckner-Hartree-Fock (BHF) approximation corresponds to the Hartree-Fock expression using the $G$ matrix for the effective interaction. This means that the self-energy of a nucleon in nuclear matter with momentum $\vec{k}$ is given by (note that spin and isospin quantum numbers are suppressed)

$$
\Sigma_{B H F}(\vec{k}, \omega)=\int d^{3} q<\vec{k} \vec{q}|G(\Omega)| \vec{k} \vec{q}>n_{0}(\vec{q}),
$$

with the occupation probability of a free Fermi Gas with a Fermi momentum $k_{F}$

$$
n_{0}(\vec{q})=\left\{\begin{array}{l}
1 \text { for }|\vec{q}| \leq k_{F} \\
0 \text { for }|\vec{q}|>k_{F}
\end{array}\right.
$$


The matrix elements in (四) denote antisymmetrized matrix elements of the Brueckner $G$ matrix which are determined by solving the Bethe-Goldstone equation for a given realistic nucleon nucleon $(\mathrm{NN})$ interaction $V$

$$
\begin{aligned}
<\vec{k} \vec{q}|G(\Omega)| \vec{k} \vec{q}>=<\vec{k} \vec{q}|V| \vec{k} \vec{q}>+ & \int d^{3} p_{1} d^{3} p_{2}<\vec{k} \vec{q}|V| \vec{p}_{1} \vec{p}_{2}> \\
& \times \frac{Q\left(p_{1} p_{2}\right)}{\Omega-\left(\tilde{\varepsilon}_{p 1}+\tilde{\varepsilon}_{p 2}\right)+i \eta}<\vec{p}_{1} \vec{p}_{2}|G(\Omega)| \vec{k} \vec{q}>.
\end{aligned}
$$

In this representation of the Bethe-Goldstone equation $Q$ stands for the Pauli operator and is defined by

$$
Q\left(p_{1} p_{2}\right)=\left(1-n_{0}\left(p_{1}\right)\right)\left(1-n_{0}\left(p_{2}\right)\right),
$$

which means that the integral over intermediate two-particle states in (3) is restricted to states with momenta larger than the Fermi momentum. According to the theorem of Bethe, Brandow and Petschek (BBP theorem) [3] one defines the single-particle energy for hole states, i.e. states with momenta $k<k_{F}$, using the on-energy shell value for the self-energy, which is given as

$$
\varepsilon_{k}=\frac{k^{2}}{2 m}+\Sigma_{B H F}\left(\vec{k}, \omega=\varepsilon_{k}\right),
$$

with a value for the starting energy parameter $\Omega$ in the Bethe-Goldstone equation (3) of

$$
\Omega=\omega+\varepsilon_{q}=\varepsilon_{k}+\varepsilon_{q} .
$$

Since this BBP theorem does not apply for the definition of the single-particle energies for states with momenta above the Fermi momentum, the optimal choice for this particle-state spectrum has been a subject of controversial discussions for many years (see the introduction above). Note that the binding energy per nucleon is evaluated as

$$
\frac{E}{A}=\frac{\int d^{3} k n_{0}(k) \frac{1}{2}\left(\frac{k^{2}}{2 m}+\varepsilon_{k}\right)}{\int d^{3} k n_{0}(k)},
$$

which implies that the definition of the particle-state spectrum affects the calculated binding energy only via the energy parameter $\tilde{\varepsilon}_{p 1}$ and $\tilde{\varepsilon}_{p 2}$ in the Bethe-Goldstone equation (3). Two choices have been discussed: the so-called "conventional" choice, in which the energies for the particle states have been replaced by the kinetic energy

$$
\tilde{\varepsilon}_{p}=\frac{p^{2}}{2 m},
$$

and the "continuous" choice, for which the on-shell definition of the hole-state energies (5) has been extended to the particle states. Note that the BHF self-energy for the continuous choice develops an imaginary part for energies $\omega>\varepsilon_{F}$ with $\varepsilon_{F}$ denoting the Fermi energy, the single-particle energy for $k=k_{F}$. The "continuous" choice, however, employs the real part of this self-energy, only. 
The on-shell definition of the BHF self-energy in eqs.(5) and (6) implies a self-consistent solution of the Bethe-Goldstone equation (3) and the evaluation of the single-particle energies. In order to obtain such a self-consistent solution one often assumes a quadratic dependence of the single-particle energy on the momentum of the nucleon in the form

$$
\varepsilon_{k} \approx \frac{k^{2}}{2 m *}+C .
$$

Starting with an appropriate choice for the parameters for the effective mass $m^{*}$ and the constant $C$, one can solve the Bethe-Goldstone equation and evaluate the single-particle energy, using (5), for two representative momenta $k_{1}$ and $k_{2}$. The parameters $m^{*}$ and $C$ are readjusted in such a way that the parametrisation (9) reproduces these two energies. This procedure is iterated until a self-consistent solution is obtained.

The parametrisation of (9), however, is useful not only to simplify the self-consistent solution of the BHF equations, it also leads to a simplification of the numerical solution of the BetheGoldstone equation. Assuming such an effective mass spectrum, one can easily rewrite the energies occurring in the denominator of the two-particle propagator in terms of the center of mass, $P_{C M}$ and relative momentum $p_{r}$ of the interacting pair of nucleons

$$
\varepsilon_{p 1}+\varepsilon_{p 2}=\frac{P_{C M}^{2}}{4 m^{*}}+\frac{p_{r}^{2}}{m^{*}}+2 C,
$$

which does not depend on the angle between $\vec{P}_{C M}$ and $\vec{p}_{r}$. If furthermore one approximates the Pauli operator of (47) by taking an appropriate average over this angle, the BetheGoldstone equation can be rewritten into a one-dimensional integral equation using the partial wave representation of the two-body states [23].

This approximation scheme has been common practise and only recently attempts have been made to avoid the angle-average approximation for the Pauli operator [8,9]. These investigations show that the use of the angle-average approximation in the Pauli operator leads to an underestimation of the calculated energies of around $0.5 \mathrm{MeV}$ per nucleon if one uses an effective mass parametrisation for the single-particle energies in the Bethe-Goldstone equation.

However, the determination of this parametrisation is not very well defined. Rather different values for $m^{*}$ and $C$ may be obtained if different momenta are chosen to define these values. This is demonstrated in Fig. 1, which displays the energy of symmetric nuclear matter at various densities, represented by the corresponding Fermi momentum. All calculations have been performed within the framework of the BHF approach but using various approximation schemes for the propagator in the Bethe-Goldstone equation. Calculations using the conventional choice for the particle-state spectrum (8) and the angle-average Pauli operator yield results, which are not very sensitive to the details of the effective mass parametrisation for the hole state energies. All resulting binding energies, which were obtained by adjusting the parameters $m^{*}$ and $C$ at different momenta, are very similar forming the thin gray area, which is labelled "conv. $m^{*}$ spectr." in Fig. 1.

The calculated binding energies are larger, if the continuous choice is employed, and the results are more sensitive to the details of the procedure to determine the parameters $m^{*}$ and $C$. This is visualised by the gray area, labelled "cont. $m^{*}$ " in Fig. 1. Both of these 
features can be related to the fact that the continuous choice spectrum tends to lead to energy denominators in the Bethe-Goldstone equation (3) with smaller absolute values than the conventional choice, which exhibits a gap in the single-particle spectrum at $k=k_{F}$.

The calculated binding energies are even larger, if the exact Pauli operator is employed and the parametrisation of the single-particle spectrum is avoided (solid line in Fig. 1). The results displayed in Fig. 11 have been obtained employing a specific version of the Bonn potential (Bonn $\mathrm{C}$ as defined in [22]). Very similar results have also been obtained using other realistic models for the NN interaction. A part of this additional binding energy can be related to the use of the exact Pauli operator (see Ref. [8] and discussion above). Another part, however, must be related to the fact that the calculated single-particle spectrum deviates in a significant way from the parametrisation of (9). Such a gain of binding energy has also recently been observed by Baldo and Fiasconaro [10 using an angle-average approach. This deviation of the self-consistent single-particle potential

$$
U_{B H F}(k)=\operatorname{Real}\left[\Sigma_{B H F}\left(\vec{k}, \omega=\varepsilon_{k}\right)\right]
$$

from its quadratic parametrisation, which is implied by (9), is explicitly displayed in Fig. 2, where results are given at two different densities using various models for the NN interaction. These models include the versions Bonn $\mathrm{A}$ and Bonn $\mathrm{C}$ of the traditional One-BosonExchange model defined in [22], the interaction models with high accuracy fits to the NN data CD Bonn [19] and Argonne V18 [20], but also the recent NN potential Idaho A [24], which is based on chiral perturbation theory.

All these single-particle potentials show a significant deviation from a parabolic shape in particular at momenta slightly above the Fermi momentum. It is obvious that such a deviation tends to provide more attractive matrix elements of $G$ in evaluating the self-energy for hole states according (1), which leads to more binding energy.

In the following we want to explore the sources of the momentum dependence of $U_{B H F}$ more in detail. For that purpose we have disentangled the dependence of the real part of the self-energy $\Sigma_{B H F}(\vec{k}, \omega)$ on energy and momentum. Following the nomenclature of Mahaux and Sartor 25] we characterise the dependence on the momentum $k$ by an effective "k-mass" $m_{k}$, which is defined by

$$
\frac{m_{k}(k)}{m}=\left[1+\frac{m}{k} \frac{\partial \Sigma_{B H F}(k, \omega)}{\partial k}\right]^{-1} .
$$

Any deviation of $m_{k}$ from the bare mass $m$ indicates a momentum-dependence of the selfenergy, which means a non-locality in coordinate space. Results for the BHF self-energy calculated for the Argonne V18 calculated in nuclear matter at a Fermi momentum $k_{F}=$ $1.36 \mathrm{fm}^{-1}$ are displayed in Fig. 3. For comparison we also include in this figure the effective $\mathrm{k}$-mass evaluated in the Hartree-Fock approximation, i.e. replacing the matrix elements of $G$ in (1) by the corresponding ones of the bare interaction $V$. One can see that the k-mass evaluated in the BHF approximation is very close to the one determined from the HartreeFock potential. This means that the non-locality of the single-particle potential originates essentially from the Hartree-Fock contribution.

The same is true for other interactions considered in this investigation. Therefore using other NN potentials we only display the effective k-mass derived from the Hartree-Fock 
approach in Fig. 3. It is remarkable that the effective k-mass derived for the traditional Bonn interaction as well as for the CD Bonn potential shows results very close to the ones derived from the V18 interaction model. This is true although the absolute values for the Hartree-Fock single-particle potentials depend quite strongly on the NN interaction, as one can see from the single-particle energies listed in the first column of table $\rrbracket$. This means that different interaction models, which all fit NN scattering data, yield quite different values for the Hartree-Fock single-particle potential in nuclear matter. The momentum dependence of these single-particle potentials, which represents the non-locality, is very similar.

In Fig. 3] it is only the Idaho A interaction model [24], as well as the version B of this model which is not contained in this figure, which shows significant deviations in particular at high momenta. These differences are due to the strong cut-off, which are employed in these interaction models. These cut-offs, which are necessary the chiral expansion at high momenta, are responsible for the fact that the Hartree-Fock potential is essentially identical to zero for momenta larger than $5 \mathrm{fm}^{-1}$.

All k-masses exhibit a rather smooth dependence on the momentum. Therefore the momentum dependence of the self-energy is not responsible for the special behaviour of the BHF self-energies at the $k=k_{F}$ displayed in Fig. 2. The energy dependence of the self-energy, so to say the non-locality in time, is characterised by the so-called "E-mass", which is defined as [25]

$$
\frac{m_{E}(\omega)}{m}=\left[1-\frac{\partial \Sigma_{B H F}(k, \omega)}{\partial \omega}\right],
$$

so that the total effective mass is given by

$$
\frac{m^{*}(k)}{m}=\frac{m_{k}(k)}{m} \frac{m_{E}\left(\omega=\varepsilon_{k}\right)}{m} .
$$

In the Hartree-Fock approximation the E-mass is identical to $m$ for all NN interaction models considered in this investigation. Therefore the deviations of $m_{E}$ from $m$, which are displayed in Fig. $⿴$ for various NN interactions originate from the ladder contributions to the G-matrix. Again we find a behaviour which is very similar for all interactions considered. The effective E-mass reaches values up to 1.4 times $m$ at energies $\omega$ slightly above the Fermi energy, which implies that the total effective mass $m^{*}$ approaches the value $m$ for momenta around the Fermi momentum, indicating that the BHF self-energy increases only weakly with $k$, just the behaviour we already observed in Fig. 2.

This energy dependence of the real part of the self-energy is also visualised in the left part of Fig. 5 displaying a pronounced minimum at energies around the Fermi energy. The right hand part of this figure shows the corresponding imaginary parts of the BHF self-energy. These imaginary parts are identical zero for energies $\omega$ less than $\varepsilon_{k}-\varepsilon_{F}$, as can be seen from eqs.(5) and (6), and yield non-negligible values up to very high energies.

Real and imaginary part of the self-energy are related to each other by a dispersion relation of the form 25,26]

$$
\operatorname{Real} \Sigma_{B H F}(k, \omega)=U_{H F}(k)+\frac{1}{\pi} \int_{-\infty}^{\infty} \frac{\operatorname{Imag} \Sigma_{B H F}\left(k, \omega^{\prime}\right)}{\omega^{\prime}-\omega} d \omega^{\prime} .
$$


Considering the results for the imaginary part of the self-energy, which are displayed in the right part of Fig. 5, it is clear from this dispersion relation that the real part of the self-energy is identical to the corresponding HF single-particle potential $U_{H F}(k)$ in the limit $\omega \rightarrow-\infty$. This real part gets more attractive with increasing $\omega$ until one reaches values of $\omega$ at which the imaginary part is different from zero. The self-energy turns less attractive at higher energies, which leads to a pronounced minimum at energies $\omega$ slightly above the Fermi energy. This energy dependence is of course also reflected in the effective E-mass discussed above.

The results obtained for the recent Idaho interactions $A$ and $B$ are quite different as can be seen from Fig. 6, which displays corresponding results for the BHF self-energy using Idaho A interaction. These differences can be traced to the strong cut-off of these Idaho interactions which have been mentioned already above. Due to these formfactors the matrixelements $<\vec{k} \vec{q}|V| \vec{p}_{1} \vec{p}_{2}>$ in the Bethe-Goldstone eq.(阿) vanish for two-body states $\mid \vec{p}_{1} \vec{p}_{2}>$ with high momenta $\vec{p}_{i}$. Therefore the imaginary part of the self-energy tends to zero for energies $\omega$ larger than $500 \mathrm{MeV}$ as shown in the left part of Fig. 6. The dispersion relation of (13) relates this shape of the imaginary part to the energy-dependence of the real part of $\Sigma_{B H F}$ leading even to positive values for $\omega \approx 400 \mathrm{MeV}$. It is worth noting that the large absolute value of imaginary part of $\Sigma_{B H F}$ obtained for the Idaho interaction at energies below 250 $\mathrm{MeV}$ yields a real part of the self-energy which is similar to the results obtained for other interactions in the energy interval for $\omega$ of $[-100 \mathrm{MeV}, 0]$, which is relevant for the BHF single-particle energies. Therefore one can expect that these Idaho interactions lead to reasonable predictions for nuclear structure at low energies and momenta. The results for processes involving nucleons with high momenta or energies will be dominated by the strong cut-off, which is required in these models to control the expansion of the chiral perturbation theory.

The different NN interactions yield quite different results for the HF single-particle potential but very similar ones for the BHF self-energy at energies $\omega$ which are close to the Fermi energy (see Fig. 5 and Table $\mathbb{Q}$ ). We can distinguish "stiff" potentials like the Argonne potential V18 or the Bonn C potential and "softer" ones like Bonn A and CD Bonn or the even softer ones Idaho A and B. The stiff potentials yield rather repulsive results for mean field of the Hartree-Fock approximation (see first column of table I). This repulsion originating from the bare $\mathrm{NN}$ interaction $V$ is compensated by the attractive particle-particle ladder contributions to the $G$-matrix. The value of this attractive contribution depends on the interaction used. It is smaller for softer potentials. If the two-particle propagator in the Bethe-Goldstone eq. (3) was replaced by the free one, i.e. no Pauli operator $Q$ and the energies in the denominator replaced by the kinetic energies, the $G$ would become identical to the T-matrix of free NN scattering. Since all potentials fit the same NN phase shifts a replacement of $G$ in the BHF equations by $\mathrm{T}$ should yield identical results. Pauli operator and the single-particle energies in the medium lead to less attractive matrix elements of $G$ as compared to $T$, which is often called Pauli- and dispersion quenching, respectively. This quenching mechanism is more efficient for the stiff potentials, since the attractive ladder contributions, which are quenched, are larger. Therefore stiff potentials lead to smaller binding energies in BHF calculations of nuclear matter as softer ones.

This can be seen from columns 1 to 3 in Table II], which lists results for the binding energy obtained in different BHF calculations of nuclear matter at three different densities, employing 
various realistic NN interactions. The dispersion quenching mechanism tends to be larger if one employs the conventional choice (8) with a gap in the single-particle spectrum for the Bethe-Goldstone equation as compared to the continuous choice. Therefore one obtains larger binding energies using the continuous choice. This is can be seen in the comparison between columns 1 and 2 of Table पII. In both kinds of calculations a parametrisation of the single-particle spectrum according to (9) has been utilised. An even larger binding energy is obtained if the exact Pauli operator and the precise shape of the single-particle energies are employed (see third column in Table [II). As discussed above this is partly due the use of the exact Pauli operator, but it is also due to the shape of the single-particle energy at $k=k_{F}$ (see Fig. 2), which is characterised by a large effective E-mass at these momenta (see Fig. 四).

\section{TOWARDS A SELF-CONSISTENT SINGLE-PARTICLE GREEN FUNCTION}

One of the arguments in favour of the continuous choice to be used in the single-particle spectrum of the Bethe-Goldstone equation has been that the corresponding definition of the single-particle self-energy would be in line with the definition of the self-energy using the method of self-consistent Green functions [2]26]. Assuming that the self-energy $\Sigma(k, \omega)$ for a nucleon with momentum $k$ and energy $\omega$ in infinite nuclear matter is given, the Dyson equation leads to a single-particle Green function of the form

$$
g(k, \omega)=\frac{1}{\omega-\frac{k^{2}}{2 m}-\Sigma(k, \omega)} .
$$

If one compares this solution with the general Lehmann representation

$$
g(k, \omega)=\lim _{\eta \rightarrow 0}\left(\int_{-\infty}^{\epsilon_{\mathrm{F}}} d \omega^{\prime} \frac{S_{\mathrm{h}}\left(k, \omega^{\prime}\right)}{\omega-\omega^{\prime}-i \eta}+\int_{\epsilon_{\mathrm{F}}}^{\infty} d \omega^{\prime} \frac{S_{\mathrm{p}}\left(k, \omega^{\prime}\right)}{\omega-\omega^{\prime}+i \eta}\right),
$$

one can easily identify the spectral functions $S_{h}(k, \omega)$ and $S_{p}(k, \omega)$ for hole and particle strength, respectively, to be given as

$$
S_{h(p)}(k, \omega)= \pm \frac{1}{\pi} \frac{\operatorname{Imag} \Sigma(k, \omega)}{\left(\omega-k^{2} / 2 m-\operatorname{Real} \Sigma(k, \omega)\right)^{2}+(\operatorname{Imag} \Sigma(k, \omega))^{2}}, \text { for } \omega<\varepsilon_{F}\left(\omega>\varepsilon_{F}\right) \text {. }
$$

The hole spectral function represents the probability that a particle with momentum $k$ and energy $\omega$ can be removed from the ground state of the system, leaving the residual nucleons in an eigenstate of the hamiltonian. The particle spectral function system contains the corresponding probability for adding a particle. In the mean field or Hartree-Fock approximation these spectral functions are reduced to $\delta$-functions of the form

$$
\begin{aligned}
& S_{p}(k, \omega)=\Theta\left(k-k_{F}\right) \delta\left(\omega-\varepsilon_{k}\right) \\
& S_{h}(k, \omega)=\Theta\left(k_{F}-k\right) \delta\left(\omega-\varepsilon_{k}\right),
\end{aligned}
$$

where the single-particle energy $\varepsilon_{k}$ contains the sum of kinetic energy plus the real part of the self-energy $\Sigma(k, \omega)$ calculated on-shell $\left(\omega=\varepsilon_{k}\right)$. 
The two-particle propagator can then be written in the form

$$
\begin{aligned}
g_{I I}\left(k_{1}, k_{2} ; \Omega\right)=\int_{\varepsilon_{F}}^{\infty} d \omega_{1} \int_{\varepsilon_{F}}^{\infty} d \omega_{2} \frac{S_{p}\left(k_{1}, \omega_{1}\right) S_{p}\left(k_{2}, \omega_{2}\right)}{\Omega-\omega_{1}-\omega_{2}+i \eta} \\
-\int_{-\infty}^{\varepsilon_{F}} d \omega_{1} \int_{-\infty}^{\varepsilon_{F}} d \omega_{2} \frac{S_{h}\left(k_{1}, \omega_{1}\right) S_{h}\left(k_{2}, \omega_{2}\right)}{\Omega-\omega_{1}-\omega_{2}-i \eta} .
\end{aligned}
$$

If one approximates the spectral functions by the mean field approximation one obtains the Galitskii-Feynman propagator, which includes particle-particle propagation as well as holehole propagation. If the hole-hole part of the propagator is ignored one obtains an equation for the ladder diagrams for the reducible two-particle Green function which corresponds to the Bethe-Goldstone equation employing the continuous choice. Using the complete Galitskii-Feynman propagator for nuclear matter at temperature $T=0$ with a realistic NN interaction leads to the so-called pairing- or deuteron-instability [11].

As a side remark we would like to mention that this instability has also been observed in studying correlation energies in finite nuclei [27]. The attempt to solve the particle-particle hole-hole RPA equations for finite nuclei in a large model space using realistic interactions and BHF single-particle energies leads to complex eigenvalues of the RPA equation. This corresponds to the pairing instability in the Galitskii-Feynman equation for infinite matter mentioned above. In finite nuclei this instability could be removed by a self-consistent single-particle propagator, which goes beyond the BHF approximation and accounts for the distribution of single-particle strength [18].

Guided by this experience in finite nuclei one can expect that the pairing instability could be removed if the single-particle Green function are evaluated in a way which account for the spectral distributions in a self-consistent way. In fact, some attempts in this direction are under investigation and first results are rather promising [28,29]. In this investigation we would like to follow a different route and calculate the contribution of the hole-hole terms to the self-energy in a kind of perturbative way [30]

$$
\Delta \Sigma_{2 h 1 p}(k, \omega)=\int_{k_{F}}^{\infty} d^{3} p \int_{0}^{k_{F}} d^{3} h_{1} d^{3} h_{2} \frac{<k, p|G| h_{1}, h_{2}>^{2}}{\omega+\tilde{\varepsilon}_{p}-\tilde{\varepsilon}_{h_{1}}-\tilde{\varepsilon}_{h_{2}}-i \eta} .
$$

In a first approximation, which we will denote as Extended Brueckner - Hartree - Fock 1 (EBHF1), we assume a single-particle spectrum $\tilde{\varepsilon}_{k}$ which Illegal variable name. is identical to the self-consistent BHF spectrum, discussed above (5), but shifted by a constant $C_{1}$, which ensures the self-consistency for $k=k_{F}$

$$
\begin{aligned}
\tilde{\varepsilon}_{k_{F}} & =\varepsilon_{k_{F}}^{B H F}+C_{1} \\
& =\frac{k_{F}^{2}}{2 m}+\Sigma_{B H F}\left(k_{F}, \omega=\tilde{\varepsilon}_{k_{F}}\right)+\Delta \Sigma_{2 h 1 p}\left(k_{F}, \omega=\tilde{\varepsilon}_{k_{F}}\right) .
\end{aligned}
$$

This shifted single-particle spectrum is also used in the Bethe-Goldstone equation.

Results for the two-hole one-particle contribution to the self-energy, $\Delta \Sigma_{2 h 1 p}$, are displayed in Fig. 7, considering various momenta $k$. The imaginary part of $\Delta \Sigma_{2 h 1 p}$ is different from zero only for energies $\omega$ below the Fermi energy. The conservation of the total momentum in the two-nucleon of the G-matrix in (19), $\vec{h}_{1}+\vec{h}_{2}=\vec{k}+\vec{p}$, leads to a minimal value of $\omega$ at 
which this imaginary part is different from zero. Due to these limitations the imaginary part integrated over all energies is much smaller for $\Delta \Sigma_{2 h 1 p}$ than for $\Sigma_{B H F}$, displayed in Fig. 5 . The real part of $\Delta \Sigma_{2 h 1 p}$ is related to the imaginary part by a dispersion relation similar to the one of (13), connecting the imaginary part of $\Sigma_{B H F}$ with the particle-particle ladder contributions to the real part of $\Sigma_{B H F}$. Since the imaginary part of $\Sigma_{2 h 1 p}$ is significantly smaller than the one of $\Sigma_{B H F}$, the same is true also for the corresponding real part.

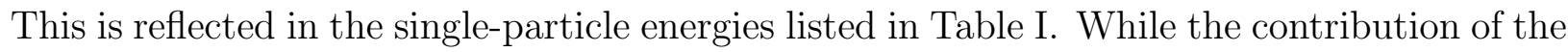
particle-particle ladders to the Fermi energy is as large as $-90 \mathrm{MeV}$ for the Bonn C or Argonne V18 interaction, the correction originating from the $2 \mathrm{~h} 1 \mathrm{p}$ term is only around $5 \mathrm{MeV}$. This is a justification of the perturbative treatment of the hole-hole ladder contribution. The non-symmetric treatment of particle-particle and hole-hole excitations, is the typical feature of the hole-line expansion. The hole-line expansions seems to be justified for nuclear systems at densities around the saturation density if one is using realistic NN interaction. The origin for this difference in the importance of particle-particle and hole-hole excitations can be read from a comparison of the imaginary parts of the self-energy contributions displayed in Fig. 5 and 7 . The phase space of particle-particle excitations, which can be excited from the ground-state by a realistic NN interaction, which is represented by an imaginary part of $\Sigma_{B H F}$ different from zero, covers a much larger energy interval than the corresponding space of hole-hole excitations (see imaginary part of $\Delta \Sigma_{2 h 1 p}$ ). The relative importance of the hole-hole contributions shall be larger at very high densities as the phase space of holehole configurations increases. The relative importance of hole-hole contributions is also larger for interactions like the Idaho A and Idaho B model, for which the particle-particle configurations which can be reached by the interaction are strongly limited by the form factors used (see Fig. 囵 and Table 凹).

The corrections of the single-particle energies due to the $\Delta \Sigma_{2 h 1 p}$ term are larger for momenta below the Fermi momentum and tend to zero for momenta above $k_{F}$. This can be seen already from Fig. 7 and is shown explicitely in Fig. 8, where the single-particle potential, i.e. the quasiparticle energy

$$
\varepsilon_{q p}(k)=\frac{k^{2}}{2 m}+\Sigma_{B H F}\left(k_{F}, \omega=\varepsilon_{q p}(k)\right)+\Delta \Sigma_{2 h 1 p}\left(k_{F}, \omega=\varepsilon_{q p}(k)\right),
$$

minus the kinetic energy is compared to the corresponding value obtained in the BHF approximation. One finds that the single-particle potential derived from the quasiparticle energy tends to a constant for momenta below $k_{F}$. This means that the effective mass which describes the momentum dependence of the quasiparticle energy $\varepsilon_{q p}$ is essentially equal to the bare mass for all momenta below $k_{F}$. At first sight one may be tempted to consider this quasiparticle spectrum also in the energy denominators of the Bethe-Goldstone equation and the $2 \mathrm{~h} 1 \mathrm{p}$ correction term of eq. (19). We will see below, however, that a different choice is more appropriate.

It is worth noting that the momentum dependence of the single-particle spectra, in the BHF as well as in the EBHF1 approximation, is very similar for all interactions considered. This means that the values obtained for $k$ smaller than the Fermi momentum using the various interactions deviate by less than $1 \mathrm{MeV}$ if they are normalised relative to the corresponding Fermi energy. Only the $\Sigma_{2 h 1 p}$ correction term is about 25 percent weaker for the Argonne V18 than for the various Bonn or Idaho interaction models (see also Fig. 8). 
From the complex self-energy $\Sigma(k, \omega)$ in the EBHF1 approach, which is the sum of the BHF term and the $2 \mathrm{~h} 1 \mathrm{p}$ correction, one can evaluate the single-particle Green function or directly the spectral functions according to (16). Results for the spectral function derived from the CD Bonn interaction for nuclear matter at the empirical saturation density are displayed in Fig. 9. For each momentum $k$ the spectral functions exhibit a maximum at the quasiparticle energy $\varepsilon_{q p}(k)$. The width of this maximum is very small for $k$ close to the Fermi momentum and gets significantly larger for very small momenta and momenta considerably larger than $k_{F}$.

More details can be seen in the logarithmic plots of the spectral function in Fig. 10. This representation also exhibits some characteristic differences depending on the interaction used. While the spectral functions derived from CD Bonn and Argonne V18 interaction exhibit a high-energy tail which extends to excitation energies $\omega$ above $1 \mathrm{GeV}$, the spectral function determined for the Idaho interaction drop very sharply at energies around 400 to $500 \mathrm{MeV}$. This is again a consequence of the strong cut-offs which are used in these interactions to control the terms in the chiral perturbation expansion.

From this figure one also observes of course that the spectral distributions are not symmetric around the quasiparticle pole. To demonstrate this on a quantitative level we calculate e.g. the mean value for the energy of the hole distribution function

$$
\overline{\varepsilon_{h}}(k)=\frac{\int_{-\infty}^{\varepsilon_{F}} d \omega \omega S_{h}(k, \omega)}{n(k)},
$$

where $n(k)$ denotes the occupation probability for the state with momentum $k$, which is calculated as

$$
n(k)=\int_{-\infty}^{\varepsilon_{F}} d \omega S_{h}(k, \omega)
$$

These mean values are significantly below the quasiparticle energies (see solid line in Fig. 8). In fact, for all interactions and densities under consideration it turned out that these results for $\overline{\varepsilon_{h}}(k)$ are close to the BHF single-particle energies for momenta $k \leq k_{F}$.

However, the mean value $\overline{\varepsilon_{h}}(k)$ is defined also for momenta larger than $k_{F}$. For those momenta we do not get a dominant contribution from the quasiparticle pole, but determine an average over a broad distribution of $2 \mathrm{~h} 1 \mathrm{p}$ configurations. Therefore the mean energies $\overline{\varepsilon_{h}}(k)$ are much more attractive for $k>k_{F}$ than for $k<k_{F}$ as it is shown in Fig. 11. As a consequence the total energy per nucleon calculated as

$$
\frac{E}{A}=\frac{\int d^{3} k \int_{-\infty}^{\varepsilon_{F}} d \omega S_{h}(k, \omega) \frac{1}{2}\left(\frac{k^{2}}{2 m}+\omega\right)}{\int d^{3} k n(k)},
$$

is significantly more attractive than the corresponding BHF result (see Table $\mathbb{I}$, column denoted EBHF1 as compared to BHF).

Fig. 11 displays in its right part also the momentum distribution $n(k)$ derived from the CD Bonn interaction (the momentum distribution for the other two Bonn potential are very similar), the Argonne V18 and the Idaho A interaction. At high momenta the result is larger for the Argonne V18 as compared to the Bonn interaction model. The Idaho interaction predicts a momentum distribution that decreases very rapidly at momenta larger than 2 
$k_{F}$. This is again a consequence of the strong cut off in this interaction and should not be considered as a realistic prediction.

The left part of Fig. 11 also shows results for the mean energy of the particle strength distribution

$$
\overline{\varepsilon_{p}}(k)=\frac{\int_{\varepsilon_{F}}^{\infty} d \omega \omega S_{p}(k, \omega)}{\int_{\varepsilon_{F}}^{\infty} d \omega S_{p}(k, \omega)}
$$

In this integration one has to include strength up to energies $\omega$ above $1 \mathrm{GeV}$, to ensure that the sum-rule

$$
n(k)+\int_{\varepsilon_{F}}^{\infty} d \omega S_{p}(k, \omega)=1
$$

is fulfilled.

For $k$ smaller than the Fermi momentum more than 80 percent of the single-particle strength is at energies below the Fermi energy. This means that the single-particle energy for these momenta might be represented by $\overline{\varepsilon_{h}}(k)$. On the other hand, for $k$ larger than $k_{F}$, the dominant part of the single-particle strength is represented by the mean energy $\overline{\varepsilon_{p}}(k)$. Therefore one might use these energies for the single-particle spectrum to be used in the propagator of the Bethe-Goldstone equation and the energy denominator of $\Delta \Sigma_{2 h 1 p}$ in (19). As one can see from Fig. 11 this would lead to a single-particle spectrum of 40 to $50 \mathrm{MeV}$ at the Fermi momentum, which is more than one half of the gap in the conventional choice for BHF. Instead of this choice we suggest a slightly different one. Since we would like to use these mean energy values to define an approximation to the single-particle Green function (15) we define a mean value $\hat{\epsilon}_{h}(k)$ for hole states by the equation

$$
\frac{1}{\hat{\epsilon}_{h}(k)-\left(\varepsilon_{F}+\frac{\Delta}{2}\right)}=\frac{1}{n(k)} \int_{-\infty}^{\varepsilon_{F}} d \omega \frac{S_{h}(k, \omega)}{\omega-\left(\varepsilon_{F}+\frac{\Delta}{2}\right)}
$$

and a corresponding one for the particle states. With this definition of a mean value one reduces in particular the contributions to the mean value for the particle states which originates from the spectral strength $S_{p}(k, \omega)$ at very high energies $\omega$ in an appropriate way. Examples for these mean values are plotted in Fig. 12. With this definition of the singleparticle spectrum one obtains a gap $\Delta$ at the Fermi energy $\varepsilon_{F}$ of about $10 \mathrm{MeV}$ for nuclear matter at saturation density. It is worth noting that this gap is of similar size as the pairing gap derived from BCS calculations for $\mathrm{T}=0$ pairing in the ${ }^{3} S_{1}-{ }^{3} D_{1}$ channel [31]. This implies that using this single-particle spectrum in a Galitzkii-Feynman equation should avoid the occurrence of pairing instabilities. Also one may mention that such a gap is in between the behaviour of the conventional and the continuous choice for the BHF spectrum discussed above.

This single-particle spectrum has then be used in the Bethe-Goldstone equation and the evaluation of the correction term $\Delta \Sigma_{2 h 1 p}$ of (19). We consider this choice to be an optimised representation of the single-particle propagator in terms of one pole (at energy $\hat{\varepsilon}(k))$ for each momentum $k$. Further improvements would require the representation of the singleparticle Green function in terms of two or more poles [17, 32] or use the complete spectral distribution. 
The calculation using the $\hat{\varepsilon}(k)$ choice is denoted by EBHF2. The self-energies, spectral functions and total energy are calculated in the same way as discussed above for the approach EBHF1. Results for the energy per nucleon are listed in the last column of Table प]. Comparing the EBHF2 results with those obtained in the EBHF1 approximation one finds that the gap in the single-particle spectrum yields a reduction of the calculated binding energy of about 1 to $3 \mathrm{MeV}$ per nucleon for the densities and interactions considered in this table. It turns out that the energies calculated in the EBHF2 approach are again close to those obtained in the BHF approximation using the exact propagator.

Our final EBHF2 results for the energy of nuclear matter derived from the Argonne V18 interaction are similar to the values determined by Akmal and Pandharipande in their variational calculation [33] using the same interaction model. The "softer" interactions like the Bonn A and the CD Bonn interaction yield larger values for the binding energy per nucleon and larger saturation densities. Even more binding energy is predicted from the recent Idaho interaction models. As it has been discussed above, these Idaho models, seem to be useful for calculations in limited model spaces only. Nevertheless, in order to obtain a result for the saturation point which is in agreement with the empirical data, some repulsive effects are needed, in particular at high densities. This repulsion can be introduced ad-hoc in terms of a three-nucleon interaction. Such an effective three-nucleon interaction may represent the effects of the relativistic decomposition of the self-energy [34] or the effects of sub-nucleonic degrees of freedom, like e.g. the many-body effects arising from $\Delta$ excitations of the nucleons [35.

\section{CONCLUSIONS}

The sensitivity of the Brueckner-Hartree-Fock (BHF) approximation for the many-body system of symmetric nuclear matter with respect to an exact treatment of the propagator in the Bethe-Goldstone equation has been investigated. One finds that the precise treatment of the Pauli operator together with a single-particle spectrum based on the real part of the self-energy for hole- and particle-states yields a result for the binding energy per nucleon which is larger by a non-negligible amount as compared to results obtained in standard approximation schemes. The non-locality and energy dependence of the BHF self-energy is discussed in detail.

The BHF definition of the self-energy has been extended to account for the effects of hole-hole ladders in a perturbative way. The corresponding results for the complex self-energy, the single-particle green function and the spectral function are discussed in detail. This leads to a definition of a spectrum of single-particle energies, which characterises the spectral distribution of the single-particle Green function in an average way. The resulting singleparticle spectrum exhibits a gap at the Fermi momentum, which is of the order of the pairing gap derived from BCS calculations for $\mathrm{T}=0$ pairing in the ${ }^{3} S_{1}-{ }^{3} D_{1}$ channel. Therefore this approximation should avoid the so-called pairing instability which occurs in the Green function approach using the Galitzkii-Feynman propagator.

The calculations have been performed employing various models for the NN interaction, which all fit NN scattering data. It is observed that the strong form factors, which had to be introduced in the recent Idaho interaction models [24] to control the expansion based on 
chiral perturbation theory, leads to results for the non-locality and spectral function, which are quite different than those obtained for the other interactions. We conclude that these new Idaho interactions should only be used for studies which are insensitive to components in the spectral distribution at higher energies or momenta.

Significant differences are also observed in comparing results between stiffer interaction models, like the Argonne V18 and Bonn C potential, or softer ones like the CD Bonn and Bonn A interaction. These differences show up in the Hartree-Fock contribution to the binding energy, which is rather repulsive for the stiff interactions and less repulsive for the softer ones. These differences can also be observed in the imaginary part of the self-energy at large energies and in the tail of the momentum distribution at high momenta.

The EBHF2 approach introduced above should be considered as a good starting point for further improvements on a self-consistent definition of the single-particle Green function. Such improvements include the representation of the Green function in terms of various poles for each momentum or attempts to account for the complete spectral distribution. The present studies demonstrate that special attention should be paid to a proper treatment of the single-particle strength around the Fermi energy.

These work has been supported by the European Graduate School "Hadrons in Vacuum, Nuclei and Stars" (Basel - T"ubingen). One of us, Kh.G., would like to thank Prof. M.M. Mustafa for useful discussions.

[1] M. Baldo, Nuclear Methods and the Nuclear Equation of State, Int. Rev. of Nucl. Physics, Vol. 9 (World-Scientific Publ. Comp., Singapore 1999).

[2] H. Müther and A. Polls, Prog. in Part. and Nucl. Phys. 45, 243 (2000) .

[3] H.A. Bethe, B.H. Brandow, and A.G. Petschek, Phys. Rev. 129, 225 (1963) .

[4] H. Kümmel, K. H. Lührmann, and J. G. Zabolitzky, Phys. Rep. 36, 1 (1978) .

[5] J.P. Jeukenne, A. Lejeune, and C. Mahaux, Phys. Rep. 25, 83 (1976) .

[6] H.Q. Song, M. Baldo, G. Gianrisacusa, and U. Lombardo, Phys. Lett. B 411, 237 (1997) ; Phys. Rev. Lett. 81, 1584 (1998) ;

M. Baldo, G. Gianrisacusa, U. Lombardo, and H.Q. Song, Phys. Lett. B 473, 1 (2000) .

[7] B.D. Day, Phys. Rev. C 24, 1203 (1981) .

[8] E. Schiller, H. Müther, and P. Czerski, Phys. Rev. C 59, 2934 (1999) ; erratum Phys. Rev. C 60, 059901 (1999) .

[9] K. Suzuki, R. Okamato, M. Kohno, and S. Nagata, Nucl. Phys. A 665, 92 (2000) .

[10] M. Baldo and A. Fiasconaro, Phys. Lett. B 491, 240 (2000) .

[11] B.E. Vonderfecht, W.H. Dickhoff, A. Polls, and A. Ramos, Nucl. Phys. A 555, 1 (1993) .

[12] T. Alm, G. Röpke, A. Schnell, N.H. Kwong, and H.S. Köhler, Phys. Rev. C 53, 2181 (1996) .

[13] P. Bożek, Nucl. Phys. A 657, 187 (1999) .

[14] M. Baldo, I. Bombaci, and U. Lombardo, Phys. Lett. B 283, 8 (1992) .

[15] T. Alm, B.L. Friman, G. Röpke, and H. Schulz, Nucl. Phys. A 551, 45 (1993) .

[16] Ø. Elgarøy, L. Engvik, E. Osnes, and M. Hjorth-Jensen, Phys. Rev. C 57, R1069 (1998) .

[17] Y. Dewulf, D. Van Neck, and M. Waroquier, Phys. Lett. B 510, 89 (2001) . 
[18] E. Heinz, H. Müther, and H.A. Mavromatis, Nucl. Phys. A 587, 77 (1995)

[19] R. Machleidt, F. Sammarruca, and Y. Song, Phys. Rev. C 53, R1483 (1996) .

[20] R.B. Wiringa, V.G.J. Stoks, and R. Schiavilla, Phys. Rev. C 51, 38 (1995) .

[21] V.G.J. Stoks, R.A.M. Klomp, C.P.F. Terheggen, and J.J. de Swart, Phys. Rev. C 49, 2950 (1994) .

[22] R. Machleidt, Adv. Nucl. Phys. 19, 191 (1989) .

[23] M.I. Haftel and F. Tabakin, Nucl. Phys. A 158, 1 (1970) .

[24] D.R. Entem and R. Machleidt, preprint nucl-th/0108057.

[25] C. Mahaux and R. Sartor, Adv. Nucl. Phys. 20, 1 (1991) .

[26] W.H. Dickhoff and H. Müther, Rep. Prog. Phys. 11, 1947 (1992) .

[27] H.A. Mavromatis, P. Ellis, and H. Müther, Nucl. Phys. A 530, 251 (1991) .

[28] W.H. Dickhoff, C.C. Gearhart, E.P. Roth, A. Polls, and A. Ramos, Phys. Rev. C 60, 064319 (1999) .

[29] W.H. Dickhoff and E.P. Roth, preprint nucl-th/0108030.

[30] P. Grange, J. Cugnon, and A. Lejeune, Nucl. Phys. A 473, 365 (1987) .

[31] U. Lombardo, P. Nozieres, P. Schuck, H.-J. Schulze, and A. Sedrakian, preprint nuclth/0109024.

[32] H. Müther, G. Knehr, and A. Polls, Phys. Rev. C 52, 2955 (1995) .

[33] A. Akmal and V.R. Pandharipande, Phys. Rev. C 56, 2261 (1997) .

[34] B.D. Serot and J.D. Walecka, Adv. Nucl. Phys. 16, 1 (1986)

[35] T. Frick, S. Kaiser, H. Müther, A. Polls, R. Entem, and R. Machleidt, preprint nucl-th/0109046

\begin{tabular}{c|ccc}
\hline \hline & HF & BHF & $+2 \mathrm{~h} 1 \mathrm{p}$ \\
\hline Bonn A & 15.66 & -37.24 & -32.48 \\
Bonn C & 60.48 & -33.53 & -29.76 \\
CD Bonn & 11.44 & -40.32 & -35.93 \\
Arg. V18 & 62.36 & -34.62 & -31.74 \\
Idaho A & -7.66 & -42.54 & -38.54 \\
Idaho B & -2.97 & -41.16 & -37.45 \\
\hline \hline
\end{tabular}

TABLE I. Fermi energy of nuclear matter with $k_{F}=1.36 \mathrm{fm}^{-1}$ calculated in the Hartree-Fock approximation (HF), the Brueckner-Hartree-Fock approximation (BHF) and with inclusion of 2 hole -1 particle contributions $(+2 \mathrm{~h} 1 \mathrm{p})$ using different interaction models. All entries are given in $\mathrm{MeV}$. 


\begin{tabular}{rc|rrrrr}
\hline \hline & $k_{F}\left[\mathrm{fm}^{-1}\right]$ & BHF, conv & BHF, cont & BHF, exact & EBHF1 & EBHF2 \\
\hline \multirow{3}{*}{ Bonn A } & 1.20 & -10.65 & -13.25 & -14.55 & -15.57 & -14.91 \\
& 1.36 & -13.39 & -15.99 & -17.46 & -18.99 & -18.13 \\
& 1.60 & -16.45 & -18.81 & -20.23 & -21.93 & -21.53 \\
\hline \multirow{3}{*}{ Bonn C } & 1.20 & -9.37 & -11.96 & -13.40 & -14.45 & -13.53 \\
& 1.36 & -11.19 & -13.82 & -15.55 & -17.03 & -15.84 \\
& 1.60 & -12.11 & -14.43 & -16.40 & -18.41 & -16.69 \\
\hline \multirow{2}{*}{ CD Bonn } & 1.20 & -11.02 & -13.79 & -15.39 & -16.19 & -15.36 \\
& 1.36 & -13.88 & -16.74 & -18.83 & -20.10 & -18.94 \\
& 1.60 & -17.00 & -19.73 & -22.86 & -24.71 & -22.81 \\
\hline \multirow{3}{*}{ Arg. V18 } & 1.20 & -9.66 & -12.03 & -13.84 & -14.65 & -13.27 \\
& 1.36 & -11.32 & -13.66 & -16.13 & -18.36 & -16.62 \\
& 1.60 & -11.65 & -14.12 & -16.77 & -19.46 & -17.09 \\
\hline \multirow{3}{*}{ Idaho A } & 1.20 & -11.91 & -14.64 & -16.14 & -17.21 & -16.55 \\
& 1.36 & -15.08 & -17.95 & -20.21 & -21.82 & -20.83 \\
& 1.60 & -18.78 & -21.88 & -25.44 & -27.62 & -26.04 \\
\hline \multirow{2}{*}{ Idaho B } & 1.20 & -11.17 & -14.10 & -15.72 & -16.75 & -15.88 \\
& 1.36 & -13.87 & -17.00 & -19.46 & -20.96 & -19.71 \\
& 1.60 & -16.55 & -19.97 & -23.94 & -25.81 & -24.07 \\
\hline \hline
\end{tabular}

TABLE II. Energy per nucleon for nuclear matter considering three different densities, $k_{F}=$ $1.20,1.36$ and $1.60 \mathrm{fm}^{-1}$. Results are displayed for BHF, assuming the conventional choice for the spectrum of particle states, BHF with a continuous choice parametrised by an effective mass, BHF using the exact Pauli operator single-particle spectrum, and for the extended BHF schemes EBHF1 as well as EBHF2 are listed for different realistic NN interactions. All energies are given in $\mathrm{MeV}$ per nucleon. 


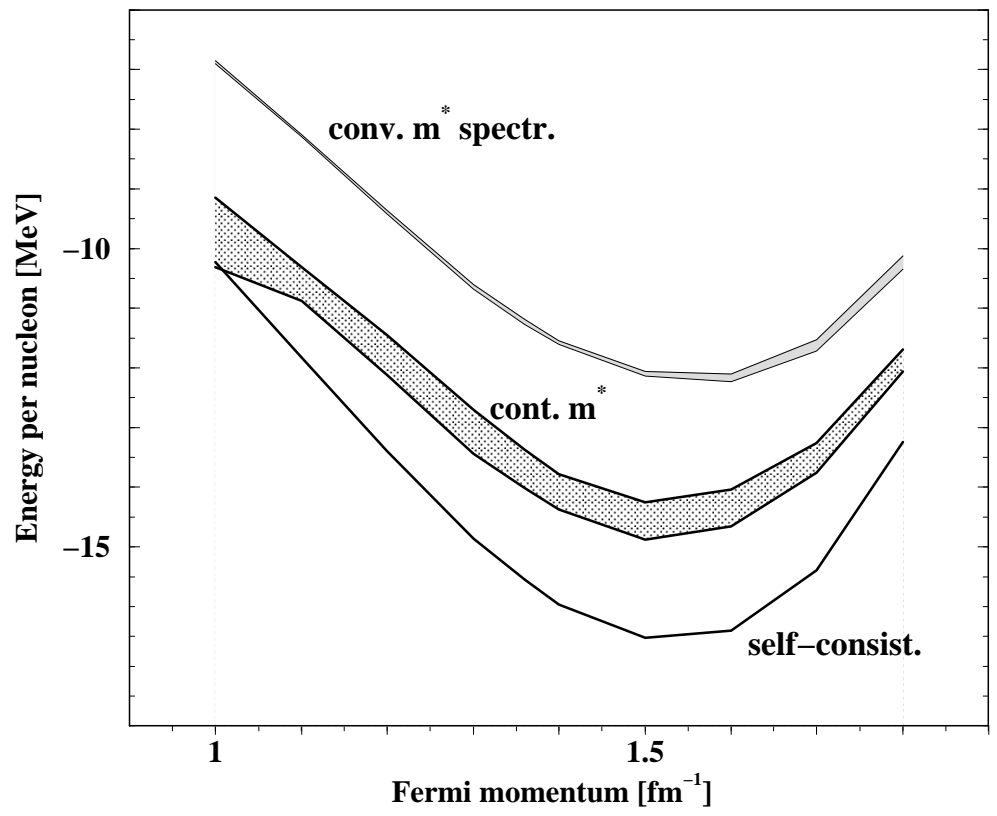

FIG. 1. Binding energy of nuclear matter as a function of the Fermi momentum. Results are given for the BHF approximation using the Bonn $\mathrm{C}$ potential as defined in [22]. Various approximation schemes have been used for the two-particle propagator in the Bethe-Goldstone equation, as discussed in the text.
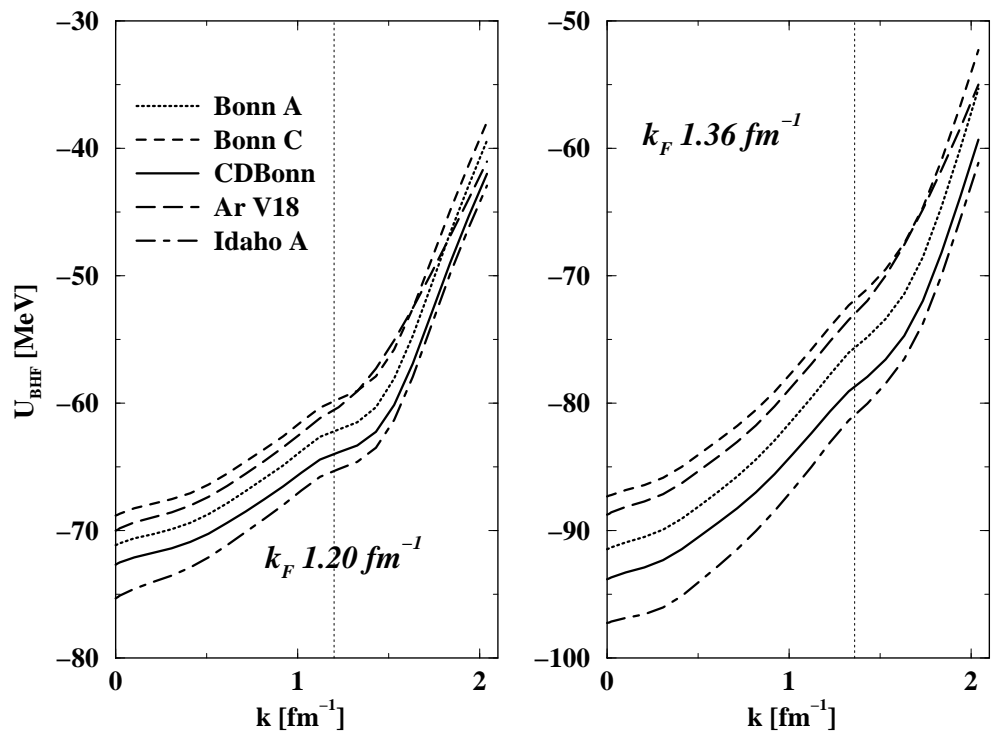

FIG. 2. The single-particle potential $U_{B H F}$ (see eq.(10)) as a function of the momentum. Results are displayed at two different densities using various models for the NN interaction. 


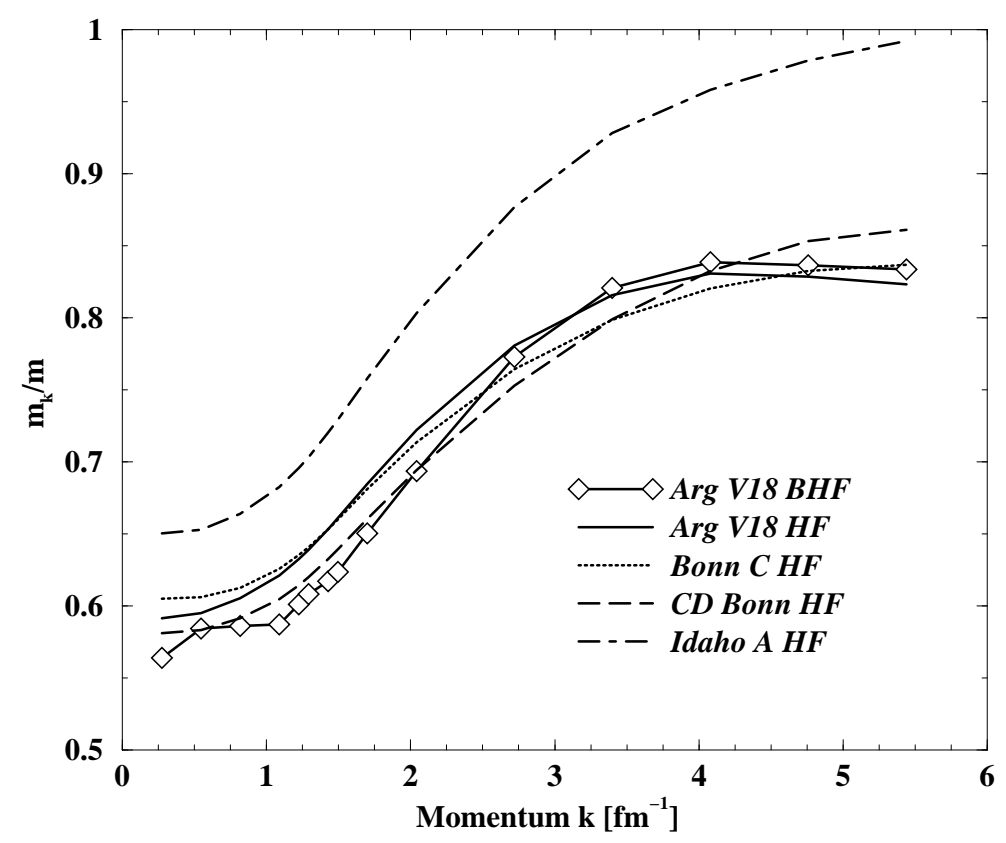

FIG. 3. The effective k-mass (see eq.(11)) evaluated for the BHF and Hartree-Fock (HF) self-energy for various interactions. Symmetric nuclear matter at the empirical saturation density $\left(k_{F}=1.36 \mathrm{fm}^{-1}\right)$ has been considered.

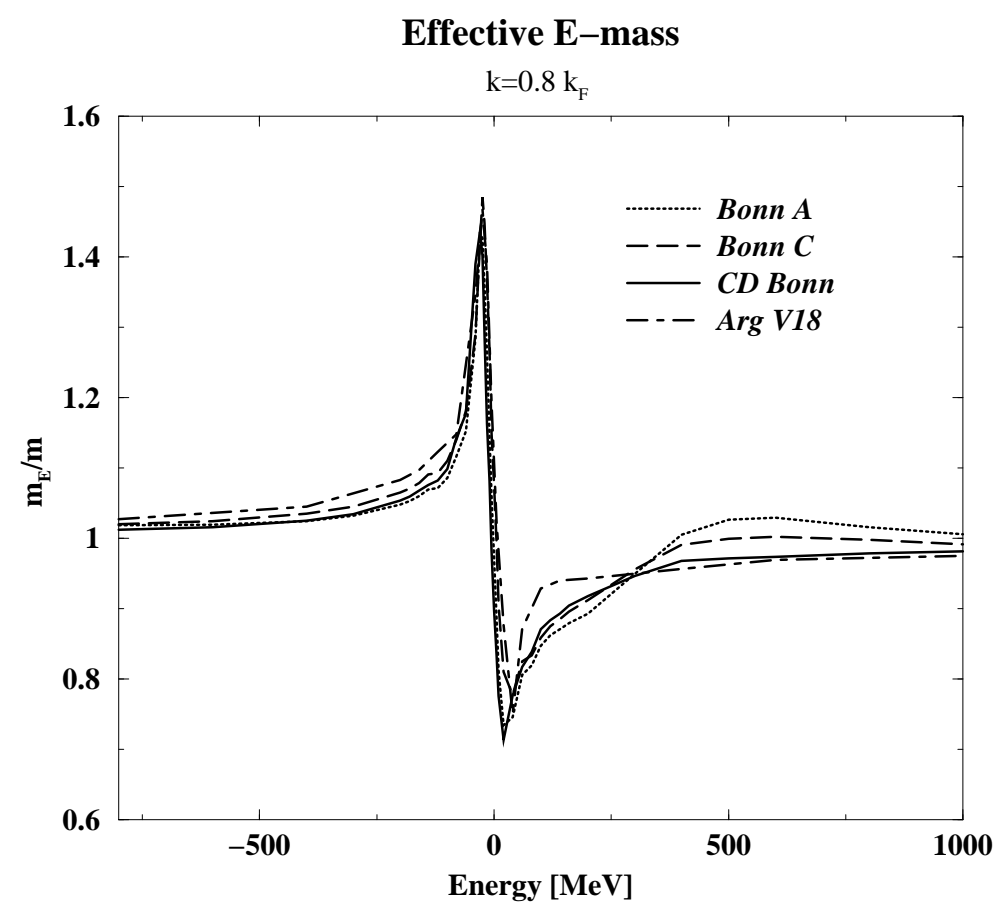

FIG. 4. The effective E-mass (see eq. (12)) evaluated for the BHF self-energy for various interactions. Symmetric nuclear matter at the empirical saturation density $\left(k_{F}=1.36 \mathrm{fm}^{-1}\right)$ has been considered, using as a typical example a momentum of $k=0.8 k_{F}$. 


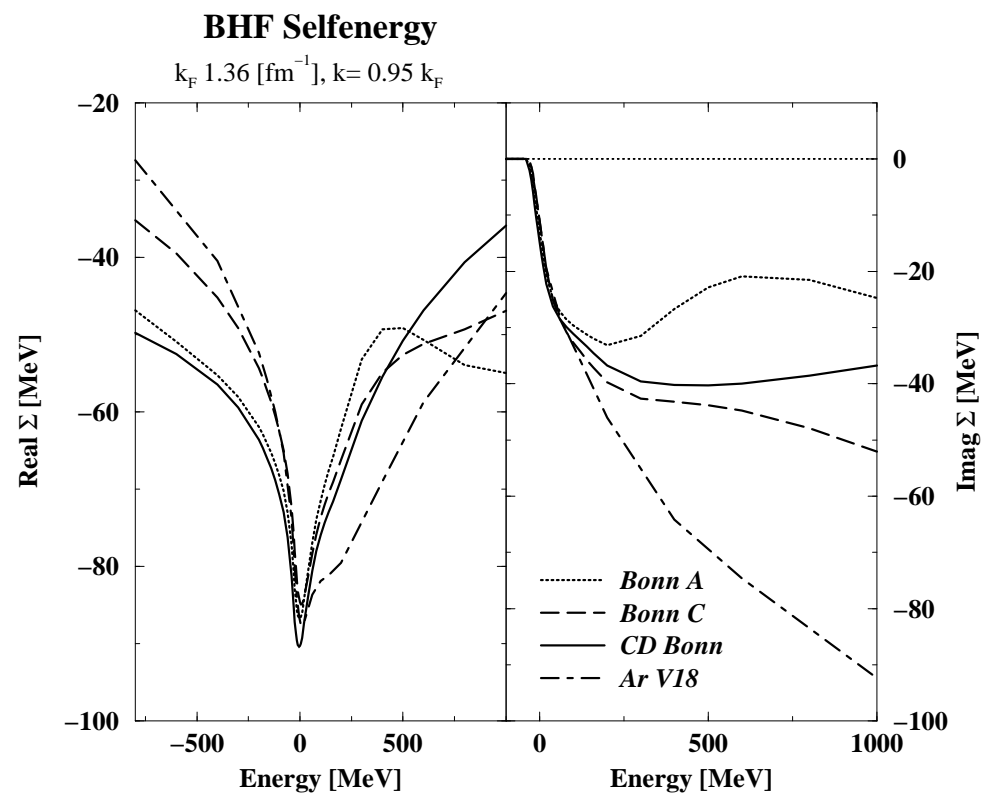

FIG. 5. The BHF self-energy (see 11) for symmetric nuclear matter with Fermi momentum $k_{F}$ $=1.36 \mathrm{fm}^{-1}$ calculated for nucleons with momentum $k=0.95 k_{F}$ as a function of the energy $\omega$. The real and imaginary part of the self-energy are displayed in right and left part of the figure, respectively. Various realistic NN interactions have been considered, which can be distinguished by the line-types as listed in the figure.

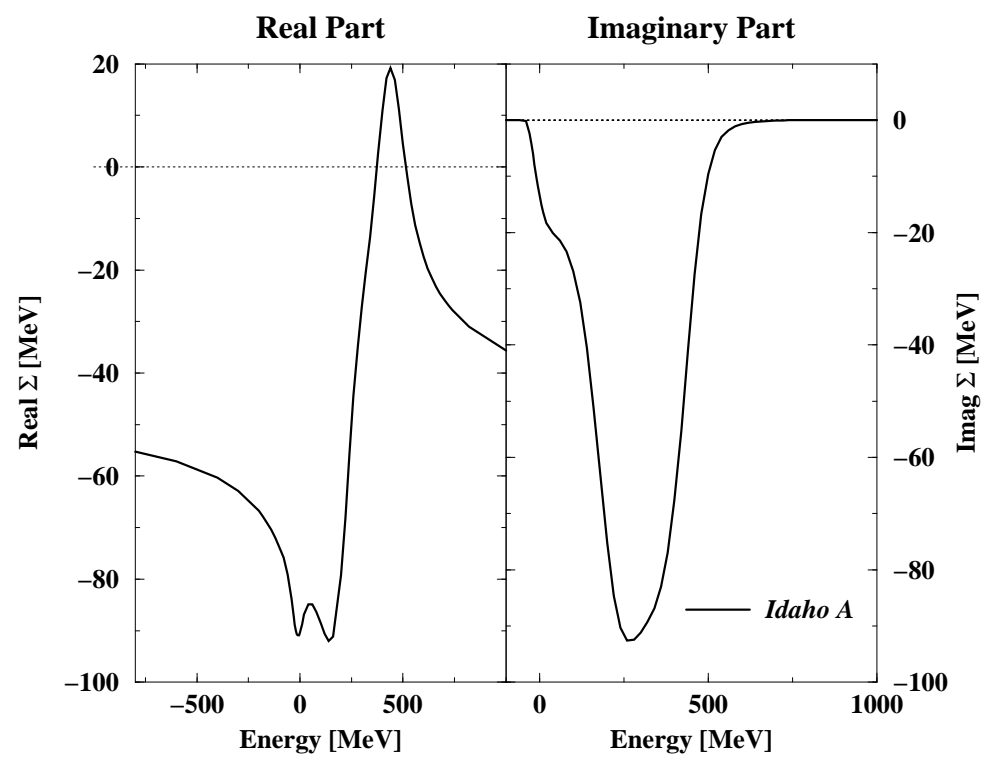

FIG. 6. The BHF self-energy (see 1) evaluated for the Idaho A interaction model. Further details as in Fig. 5 


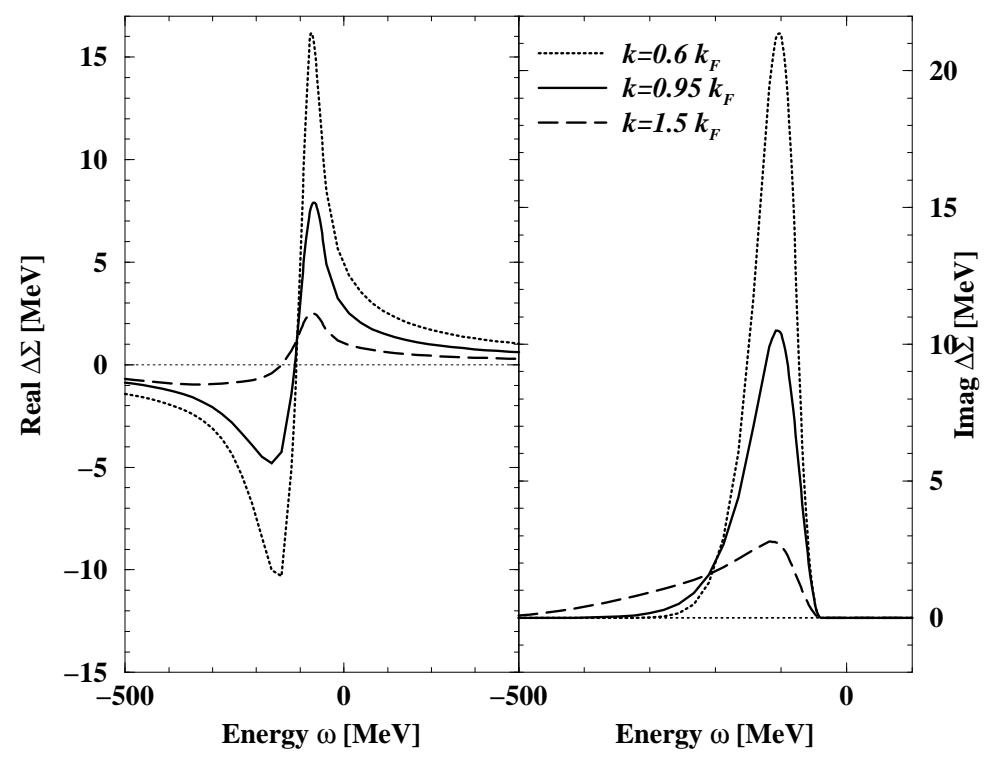

FIG. 7. The $2 \mathrm{~h} 1 \mathrm{p}$ contribution to the self-energy (see 19) evaluated for the CD-Bonn interaction assuming $k_{F}=1.36 \mathrm{fm}^{-1}$ calculated for various momenta $k$. Real (left part) and imaginary part(right part of the figure) are displayed as function of $\omega$. 


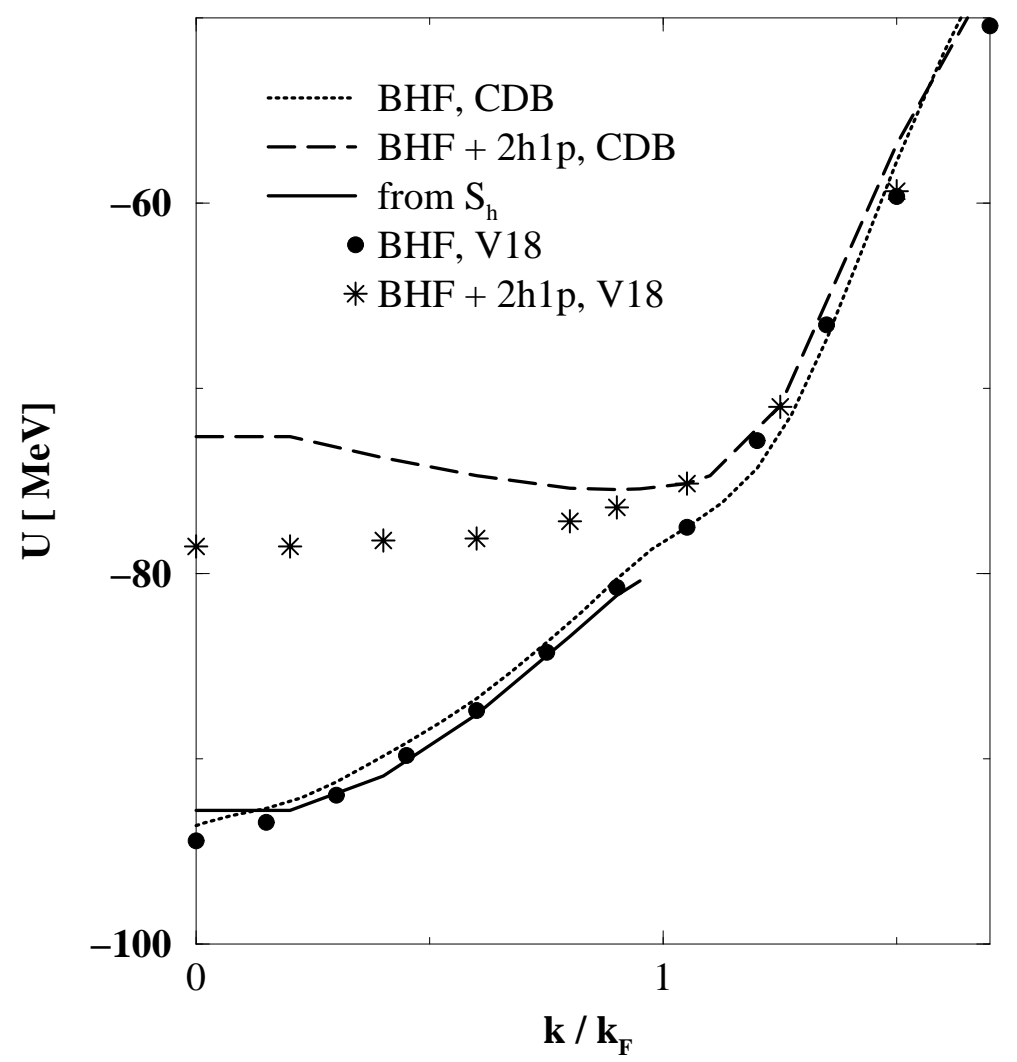

FIG. 8. The single-particle potential, i.e. the single-particle energy minus the kinetic energy, as a function of the momentum assuming the BHF approximation, the quasiparticle energy originating from the $\mathrm{BHF}+2 \mathrm{~h} 1 \mathrm{p}(\mathrm{EBHF} 1)$ approximation and the mean value derived from the hole spectral function according to (22). The data have been obtained for nuclear matter with a Fermi momentum $k_{F}=1.36 \mathrm{fm}^{-1}$, assuming the CD Bonn potential and the Argonne V18. The results for the V18 interaction have been shifted by a constant such that the BHF results for $k=k_{F}$ agree with those derived from the CD Bonn potential. 


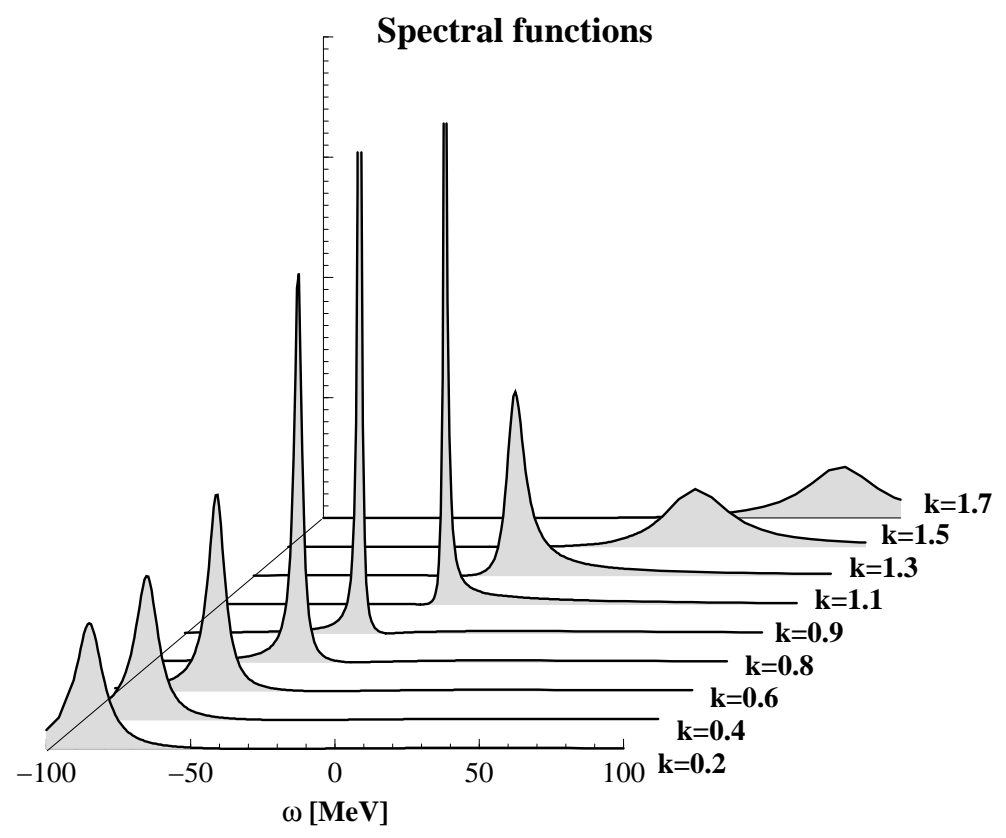

FIG. 9. The spectral function for particle and hole strength $S_{h}(k, \omega)+S_{p}(k, \omega)$ as a function of energy assuming various momenta. The data have been obtained for nuclear matter with a Fermi momentum $k_{F}=1.36 \mathrm{fm}^{-1}$, assuming the CD Bonn potential. 


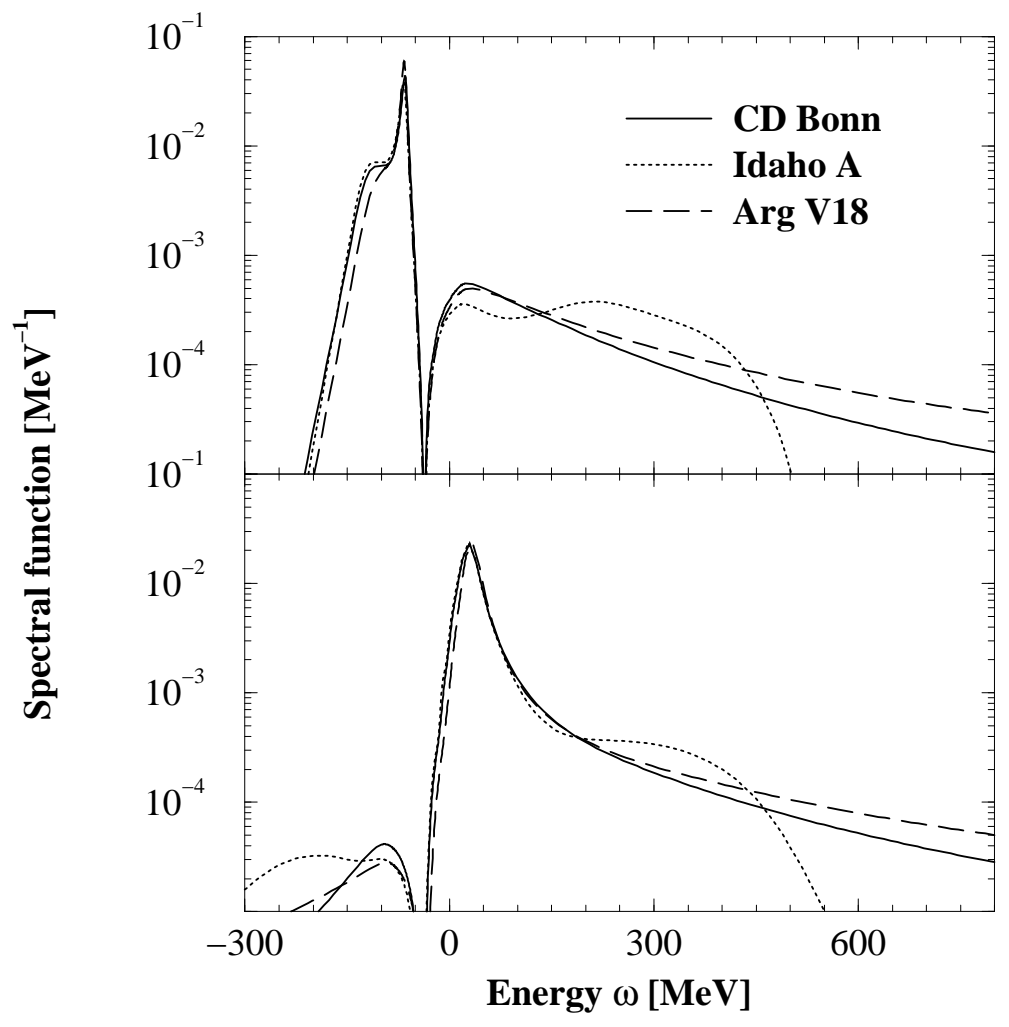

FIG. 10. The spectral function for particle and hole strength $S_{h}(k, \omega)+S_{p}(k, \omega)$ as a function of energy assuming $k=0.4 k_{F}$ (upper part of the figure) and $k=1.5 k_{F}$ (lower half of the figure). The data have been obtained for nuclear matter with a Fermi momentum $k_{F}=1.36 \mathrm{fm}^{-1}$, assuming three different $\mathrm{NN}$ interactions. 


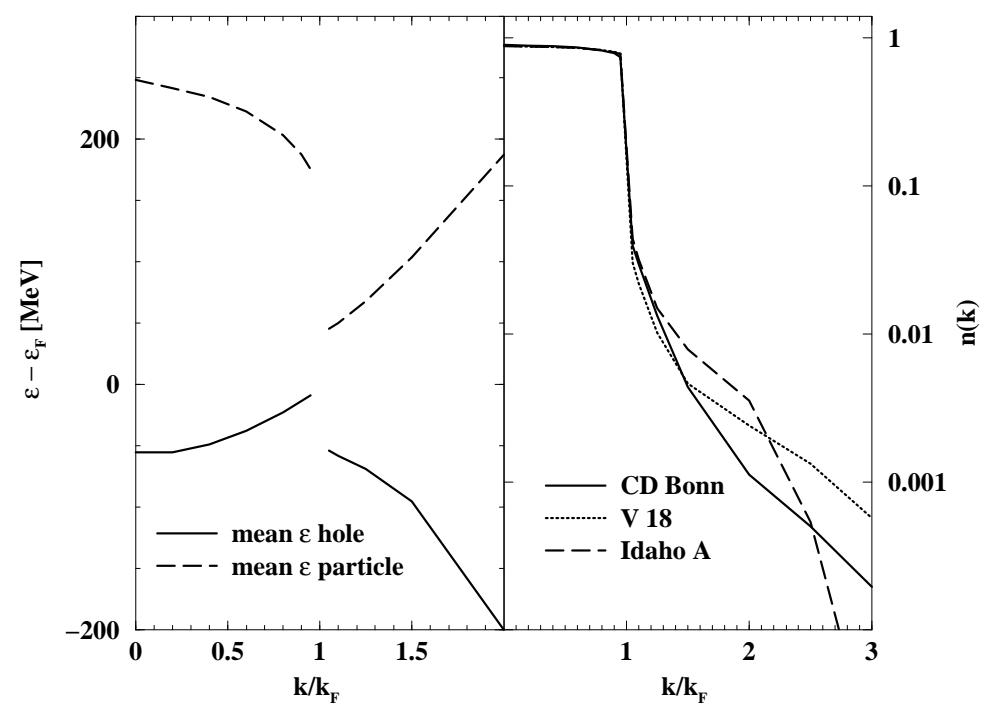

FIG. 11. The left part of the figure exhibits the mean value for the single-particle energy weighted by the spectral function for particle and hole strength, respectively, relative to the Fermi energy. Results are given for the example of the Bonn $\mathrm{C}$ potential. The part on the right-hand side shows the occupation probability $n(k)$ for various interaction. All results in this figure refer to nuclear matter with a Fermi momentum $k_{F}=1.36 \mathrm{fm}^{-1}$. 


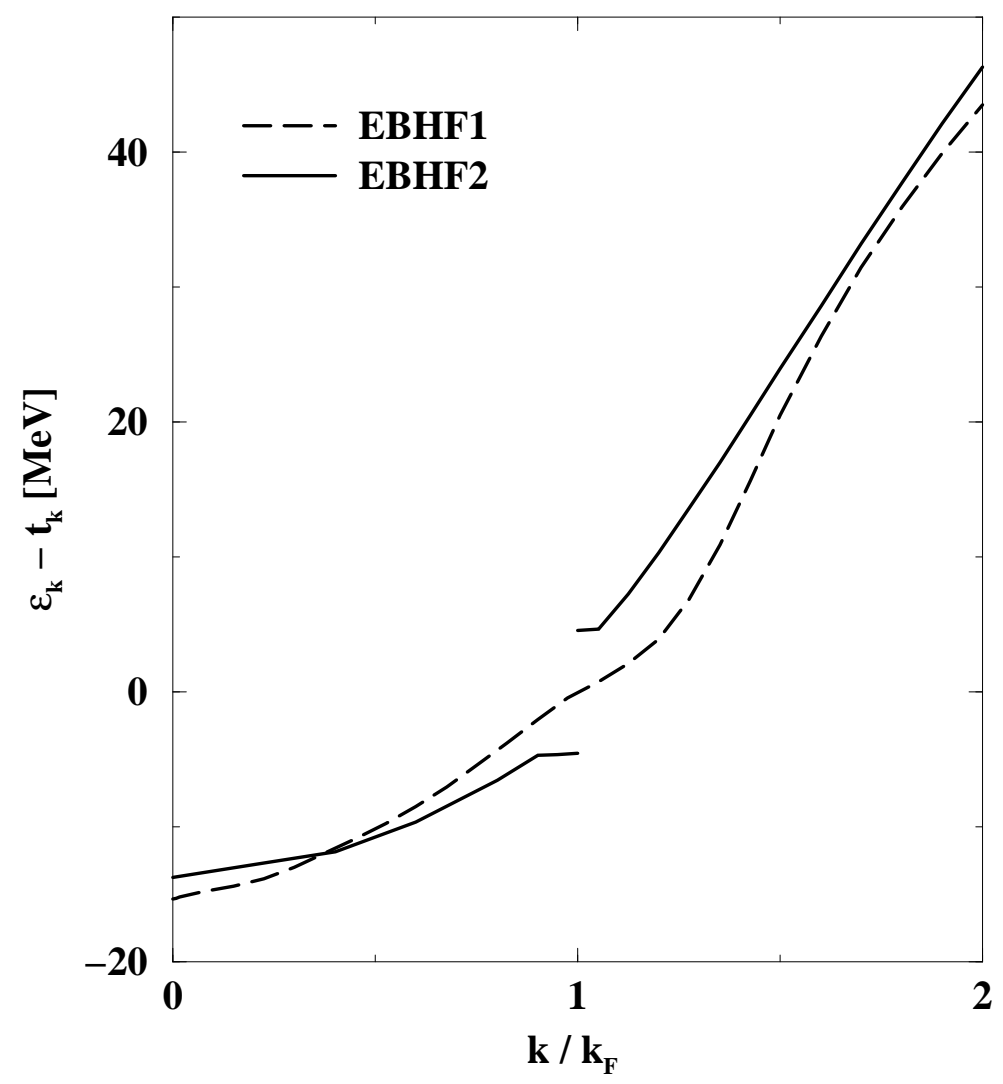

FIG. 12. The mean value $\hat{\epsilon}_{h}(k)$ defined in eq. (26) and the corresponding $\hat{\epsilon}_{p}(k)$ (solid lines) are compared to the single-particle spectrum obtained within the BHF approximation (dashed line). Note that the kinetic energies have been subtracted and the curves have been shifted to obtain the value 0 for $k=k_{F}$. The results in this figure refer to nuclear matter with a Fermi momentum $k_{F}$ $=1.36 \mathrm{fm}^{-1}$ and have been derived from the CD Bonn interaction. 